\title{
THE TANGENT BUNDLE OF AN $H$-MANIFOLD
}

\author{
JEROME KAMINKER
}

\begin{abstract}
By an $H$-manifold we mean a closed, smooth $\left(C^{\infty}\right)$ manifold which is an $\mathrm{H}$-space. It is proved that the tangent sphere bundle of an $H$-manifold is fiber homotopy equivalent to the trivial bundle. This improves a result of $W$. Browder and $E$. Spanier which proved only the stable fiber homotopy triviality. As an application, we observe that a 1-connected, finite, $\mathrm{CW}$ complex, which is an $\boldsymbol{H}$-space (and, hence, an $\boldsymbol{n}$-dimensional Poincaré complex, for some $n$ ) is of the homotopy type of a parallelizable manifold, if $n \neq 4 k+2$.
\end{abstract}

1. Introduction. Let $M^{m}$ be a closed, smooth, $m$-dimensional manifold which admits an $H$-space product, $m: M \times M \rightarrow M$, with unit $e$. We will call $M$ an $H$-manifold. In [4], W. Browder and E. Spanier prove that the tangent sphere bundle of $M, T \circ M$, is stably fiber homotopy trivial. The purpose of this note is to prove that $T_{\circ} M$ is actually fiber homotopy trivial.

This is the strongest assertion one can make in general, in the following sense: there is an example of an $H$-manifold, $M$, with a nonzero rational Pontrjagin class [1]. This means that $T M$ is not even stably trivial as a Euclidean bundle [14].

2. Main results. In [11], J. Wagoner and $\mathrm{R}$. Benlian prove that the fiber homotopy type of the tangent sphere bundle of a smooth manifold is a homotopy type invariant.

We observe, first, that the techniques of [11], also prove the following:

THEOREM 1 (WAgONER AND BENLIAN). Let $W^{k}$ and $N^{n}$ be closed, smooth manifolds, with $2 n \leqq k$, and $n \geqq 3$. Let $f$ and $g$ be embeddings of $N$ into $W$. Suppose there is a homotopy equivalence, $F: W \rightarrow W$, such that $F f \simeq g$.

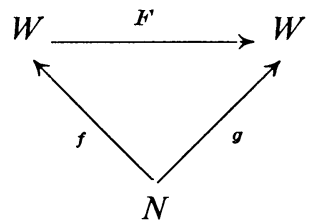

Received by the editors January 5, 1972 and, in revised form, February 5, 1973.

AMS (MOS) subject classifications (1970). Primary 55D45, 57D15.

Key words and phrases. $H$-space, fiber homotopy type, tangent sphere bundle, parallelizable manifold.

(c) American Mathematical Society 1973 
Assume, further, that $f$ and $g$ induce monomorphisms on the fundamental group. Then the normal sphere bundles of $f$ and $g$ are fiber homotopy equivalent.

Proof. (For completeness, we state the two main ideas of the proof. The details of the proof are identical with those of Proposition 1 of [11].) First one gets, via codimension one surgery [12], and an embedding theorem of Haefliger [8], an $h$-cobordism $V^{k+1}$, with $\partial V=W \cup W_{1}$, and an embedding $g_{1}: N \rightarrow W_{1}$, such that $f$ is homotopic to $g_{1}$ in $V$. By the methods of [9], it is shown that the normal sphere bundles of $g_{1}$ and $g$ are fiber homotopy equivalent.

The second step is to observe that $f$ and $g_{1}$ extend to an immersion of $N \times I$ into $V$, so that their normal sphere bundles are equivalent. This is shown by putting the homotopy between $f$ and $g_{1}$ into general position and using a theorem of Whitney [13], to redefine it in neighborhoods of the singular points, so that the new homotopy is an immersion of $N \times I$ in $V$. We refer the reader to [11] for a more complete version.

The main result now follows easily.

THEOREM 2. Let $M^{m}$ be a connected, closed, smooth H-manifold. Then the tangent sphere bundle of $M$ is fiber homotopy trivial.

Proof. We may assume $m \geqq 3$, since the only closed connected $H$ manifolds of dimension 1 or 2 are $S^{1}$ and $S^{1} \times S^{1}$, and they are parallelizable. Let $m: M \times M \rightarrow M$ be an $H$-space product, with unit $e$. Apply Theorem 1, with $W=M \times M, f: M \rightarrow M \times M$ defined by $f(x)=(x, e), g: M \rightarrow M \times M$ the diagonal map, and $F: M \times M \rightarrow M \times M$ defined by $F(x, y)=(x, m(x, y))$. Note that the dimension hypotheses of Theorem 1 are satisfied, and $F$ is a homotopy equivalence [15]. Moreover, $F f=g$ and, both $f$ and $g$ induce monomorphisms on the fundamental group. Therefore, the normal sphere bundles of $f$ and $g$ are fiber homotopy equivalent. But, the normal bundle of $g$ is the tangent bundle of $M$, and the normal bundle of $f$ is trivial.

3. Remarks. (a) According to [4], [7], and [10], there is a single obstruction to the fiber homotopy triviality of the tangent sphere bundle of an $H$-manifold. In even dimensions it vanishes since the Euler characteristic of a closed $H$-manifold is zero (unless it is a point) [10]. In odd dimensions, the obstruction vanishes if the mod 2 Kervaire semicharacteristic of $M$

$$
\chi^{*}(M) \equiv \sum_{i=0}^{[k / 2]} \operatorname{dim} H^{i}\left(M ; Z_{2}\right) \bmod 2,
$$


is zero [7]. The geometric proof in Theorem 1 and Theorem 2 show this to be the case.

(b) If $M$ is 1-connected, with dimension greater than five, and an $H$-space, but only a PL or topological manifold, then the tangent microbundle of $M$ is fiber homotopy trivial.

4. Application. Although it is known that a finite $\mathrm{CW}$ complex, $X$, which is an $H$-space, is not necessarily homotopy equivalent to a compact Lie group, we can use Theorem 2 to get a reasonably nice representative of its homotopy type.

According to [2], there exists an $n$ such that $X$ is an $n$ dimensional Poincaré complex. From [4], we see that the product vector bundle over $X$ is reducible and is therefore stably fiber homotopy equivalent to the Spivak normal fibration. In this situation, simply connected surgery yields:

Theorem 3. Let $X$ be a 1-connected finite H-complex. Suppose $X$ has dimension $n$ as a Poincaré complex, and $n \neq 4 k+2$. Then $X$ is of the homotopy type of a parallelizable manifold.

Proof. First assume $n \geqq 5$. It will follow from [3] that $X$ is homotopy equivalent to a $\pi$-manifold, $M$, if the surgery obstruction vanishes when $n=4 k$. But this is equivalent to the index of $X, I(X)$, being zero, which is so because $X$ is an $H$-space [6]. (This can be seen easily-for $I(X)=$ $I\left(S_{1} \times \cdots \times S_{l}\right)$, each $S_{j}$ an odd dimensional sphere. But the index is multiplicative and zero on odd dimensional manifolds.) Thus, the obstruction vanishes. By Theorem 2, the tangent sphere bundle of $M$ is fiber homotopy trivial. By Sutherland [10], this is sufficient to make $M$ parallelizable.

For $1 \leqq n \leqq 4$, we note that $X$ will be a 1 -connected finite complex with vanishing Euler characteristic. None can exist in dimensions 1 and 2. In dimension 3 or $4, X$ would be a homology sphere. But a homology 4-sphere has the wrong cohomology for an $H$-space, and by Browder [5], a 1-connected homology 3-sphere is of the homotopy type of $S^{3}$, a parallelizable manifold.

\section{BIBLIOGRAPHY}

1. V. Belfi, Nontangential homotopy equivalences, Pacific J. Math. 36 (1971), 615-621. MR 43 \#8086.

2. W. Browder, Torsion in H-spaces, Ann. of Math. (2) 74 (1961), 24-51. MR 23 \#A2201.

3. - Surgery on simply connected manifolds, Mimeographed, 1969.

4. W. Browder and E. Spanier, H-spaces and duality, Pacific J. Math. 12 (1962), 411-414. MR 26 \#1891. 
5. W. Browder, Higher torsion in H-spaces, Trans. Amer. Math. Soc. 108 (1963), 353-375. MR 27 \#5260.

6. - Homotopy type of differentiable manifold, Aarhus Colloqium, 1962.

7. J. Dupont, On homotopy invariance of the tangent bundle. I, Math. Scand. 26 (1970), 5-13. MR 42 \#8516.

8. A. Haefliger, Plongements différentiables de variétés dans variétés, Comment. Math. Helv. 36 (1961), 47-82. MR 26 \#3069.

9. M. W. Hirsch, On the fibre homotopy type of normal bundles, Michigan Math. J. 12 (1965), 225-229. MR 32 \#1724.

10. W. Sutherland, Fiber homotopy equivalence and vector fields, Proc. London Math. Soc. (3) 15 (1965), 543-556. MR 31 \#763.

11. J. Wagoner and R. Benlian, Type d'homotopie fibré et réduction structurale des fibrés vectoriels, C. R. Acad. Sci. Paris, Sér. A-B 265 (1967), A207-A209. 36 \#4576.

12. C. T. C. Wall, Surgery on compact manifolds, Academic Press, New York, 1970.

13. $\mathrm{H}$. Whitney, The singularities of a smooth n-manifold in $(2 n-1)$ space, Ann. of Math. (2) 45 (1944), 247-293. MR 5, 274.

14. J. Schafer, Topological Pontrjagin classes, Comment. Math. Helv. 45 (1970), 315-332. MR 43 \#1199.

15. M. Sugawara, On a condition that a space is an H-space, Math. J. Okayama Univ. 6 (1957), 109-129. MR 19, 160.

Department of Mathematics, Indiana University, Bloomington, Indiana 47401 